ARTICLE

\title{
Nanocrystal facet modulation to enhance transferrin binding and cellular delivery
}

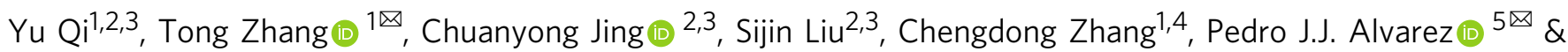
Wei Chen ${ }^{1}$

Binding of biomolecules to crystal surfaces is critical for effective biological applications of crystalline nanomaterials. Here, we present the modulation of exposed crystal facets as a feasible approach to enhance specific nanocrystal-biomolecule associations for improving cellular targeting and nanomaterial uptake. We demonstrate that facet-engineering significantly enhances transferrin binding to cadmium chalcogenide nanocrystals and their subsequent delivery into cancer cells, mediated by transferrin receptors, in a complex biological matrix. Competitive adsorption experiments coupled with theoretical calculations reveal that the (100) facet of cadmoselite and (002) facet of greenockite preferentially bind with transferrin via inner-sphere thiol complexation. Molecular dynamics simulation infers that facet-dependent transferrin binding is also induced by the differential affinity of crystal facets to water molecules in the first solvation shell, which affects access to exposed facets. Overall, this research underlines the promise of facet engineering to improve the efficacy of crystalline nanomaterials in biological applications.

\footnotetext{
${ }^{1}$ College of Environmental Science and Engineering, Ministry of Education Key Laboratory of Pollution Processes and Environmental Criteria, Tianjin Key Laboratory of Environmental Remediation and Pollution Control, Nankai University, 38 Tongyan Rd., Tianjin 300350, China. ${ }^{2}$ State Key Laboratory of Environmental Chemistry and Ecotoxicology, Research Center for Eco-Environmental Sciences, Chinese Academy of Sciences, Beijing 100085, China. ${ }^{3}$ University of Chinese Academy of Sciences, Beijing 100049, China. ${ }^{4}$ School of Environment, Beijing Normal University, 19 Xinjiekouwai St., Beijing 100875, China.


alvarez@rice.edu
} 
C rystalline nanomaterials can be conjugated with a variety of biomolecules, including peptides, proteins (e.g., antibodies and enzymes), nucleic acids (e.g., aptamers), and lipids, for enhancing their biocompatibility and in vivo circulation, as well as for enabling cell recognition and intracellular delivery ${ }^{1-5}$. This approach is becoming increasingly prominent for diagnosis and treatment of cancer, anemia, diabetes, and Alzheimer's disease via nano-enabled biosensors, bioimaging, and drug delivery ${ }^{6-8}$. Significant attention has focused on modulating the interaction between crystalline nanomaterials and biomolecules through manipulating the morphology, particle size, and surface functionalities of nanocrystals ${ }^{9-12}$, whereas exposed crystal facets, one of the most intrinsic properties of crystalline nanomaterials, remain largely unexplored.

Some biomolecules are known to exhibit differential affinities toward dissimilar crystal surfaces. For example, thermal hysteresis proteins, a group of serum proteins commonly present in organisms living in cold environments, bind to specific faces of ice crystals to enable their antifreeze activity ${ }^{13,14}$. Recent theoretical studies point to the possibility of facet-dependent selective binding of amino acids, peptides, proteins, and DNA to crystal surfaces containing metals ${ }^{15-19}$. Here, we experimentally prove the concept that facet engineering may be utilized to tune the nanocrystal-biomolecule association for refining biological applications of crystalline nanomaterials. Using transferrinfacilitated cellular targeting as a model system (which has been widely applied in cancer-related research ${ }^{20-22}$ ), we demonstrate that cadmium chalcogenide nanocrystals with specific facets (i.e., (100) facet of cadmoselite and (002) facet of greenockite) preferentially bind with transferrin via inner-sphere coordination in a complex protein matrix, which significantly enhances receptor-mediated delivery of the nanocrystals into cancer cells.

\section{Results}

Characterization of different-faceted nanocrystals. Three types of facet-engineered cadmium chalcogenide nanocrystals were used in this study, including cadmium selenide (CdSe) nanoparticles (CdSe-p), CdSe nanorods (CdSe-r), and cadmium sulfide (CdS) nanorods (CdS-r). The crystalline phase of CdSe and $\mathrm{CdS}$ was cadmoselite and greenockite, respectively. For each type of nanocrystals, two materials (denoted as " $A$ " and "B") with different content of exposed facets were synthesized to exhibit similar size and morphology. The relative height of the (100) or (101) vs. (002) peaks in the X-ray diffraction (XRD) spectra was used to estimate the relative content of these facets in each material ${ }^{23}$. CdSe-p-A and CdSe-r-A had larger relative content of (100) compared with the corresponding " $\mathrm{B}$ " materials, while CdS-r-A had larger relative content of (002) compared with CdSr-B (Fig. 1a-c). CdSe nanoparticles appeared to be spherical particles with diameter of $\sim 30 \mathrm{~nm}$ (Fig. 1d), while the dimensions of CdSe and CdS nanorods were $400 \times 20 \mathrm{~nm}$ (Fig. 1e) and $100 \times$ $10 \mathrm{~nm}$ (Fig. 1f), respectively. The hydrodynamic diameter, $\xi$ potential and Brunauer-Emmett-Teller (BET) surface area of the " $A$ " materials were similar to those of the respective " $B$ " materials (Supplementary Table 1). Thus, the difference in exposed crystal facets was the main factor determining differences in nanocrystal-transferrin binding efficacy and uptake by cancer cells.

Facet-dependent transferrin binding to nanocrystals. The facetengineered cadmium chalcogenide nanocrystals were incubated in a model protein matrix (i.e., fetal bovine serum, FBS) that contained transferrin along with a diverse group of proteins. The composition of the hard protein corona on CdSe-p, CdSe-r, and CdS-r was analyzed using liquid chromatography-mass spectrometry/mass spectrometry (LC-MS/MS, Supplementary Data). The exponentially modified protein abundance index (emPAI) ratio of the protein fractions associated with the nanocrystals with respect to the fractions in FBS (i.e., enrichment factor $^{24}$ ) was calculated to represent the degree of enrichment of specific proteins in the hard corona. Interestingly, transferrin was the most enriched protein in the corona on all the tested nanocrystals, particularly for the " $\mathrm{A}$ " materials, as indicated by the larger enrichment factor of transferrin compared with the other serum proteins (Fig. 2; Supplementary Data). Moreover, among all the proteins in the matrix, transferrin exhibited the greatest difference in the enrichment factor of the " $A$ " vs. " $B$ " samples (Fig. 2; Supplementary Data). These results indicate that $\mathrm{Cd}$ nanocrystals bind transferrin preferentially in the presence of other biomolecules, and this binding process is susceptible to facet modulation, which may be exploited to enable specific binding of transferrin for enhanced cell targeting of nanocrystals in a complex and realistic biological matrix.

Facet-dependent delivery of nanocrystals into cancer cells. Indeed, both single-cell-inductively coupled plasma-mass spectrometry (SC-ICP-MS, Fig. 3a-d) and confocal fluorescence microscopy analysis (Fig. 3e) of HeLa cells after exposed to the transferrin-CdSe conjugates (transferrin was FITC-labeled for confocal fluorescence microscopy) revealed that the stronger binding between transferrin and CdSe-p-A, relative to CdSe-p-B, resulted in greater uptake of these protein-nanocrystal conjugates into HeLa cells. This facet-dependent cellular uptake was mediated via transferrin receptors, proven by the endocytosis experiments using HeLa cells with transferrin receptors silenced by small interfering RNA (siRNA). The siRNA-transfected cells assimilated much less nanomaterial with no apparent differences between CdSe-p-A and CdSe-p-B (Supplementary Fig. 1). Note that previous research has shown that transferrin-nanomaterial conjugates may lose the targeting function due to the formation of a corona by other biomolecules (abundant in biological environments) on nanomaterial surfaces that hinder the intended function $^{11}$. Our results clearly demonstrate that transferrin molecules that preferentially associated with CdSe nanocrystals with more abundant (100) facet remained active after adsorption, and were recognized by the receptors on the HeLa cells.

\section{Discussion}

Effective application and optimization of this facet-facilitated cell targeting phenomenon calls for mechanistic understanding of the surficial interaction between transferrin and different exposed facets of the cadmium chalcogenide nanocrystals. Nanoparticles that contain soft metals (e.g., $\mathrm{Au}, \mathrm{Zn}$ ) are known to have strong affinity for and tend to adsorb thiol-containing ligands by forming coordination bonds ${ }^{25,26}$. Given that inner-sphere complexation has been shown to be an important mechanism for facet-dependent adsorption of anion ligands on hematite nanocrystals ${ }^{27,28}$, it is reasonable to postulate that the facetdependent binding between transferrin, a thiol-rich protein, and nanocrystals containing soft metal Cd is likely controlled by the metal-thiol complexation process. Accordingly, we demonstrate that the relatively high thiol content is an important factor controlling the facet-dependent preferential binding of transferrin to cadmium chalcogenide nanocrystals by substituting all cysteine for glycine to synthesize a non-thiol mutation of transferrin (Fig. 4a) and comparing this mutation with the thiol-rich transferrin (Cys\% 5.39, Fig. 4b) in adsorption experiments. In all cases (i.e., CdSe-p, CdSe-r, and CdS-r), thiol-rich transferrin preferentially bound with the " $A$ " rather than the " $B$ " materials (Fig. 4c-e), consistent with the trend in proteomic analysis 
a
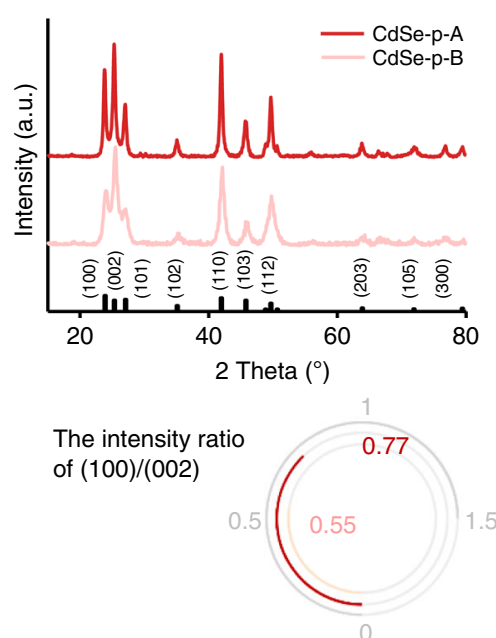

d
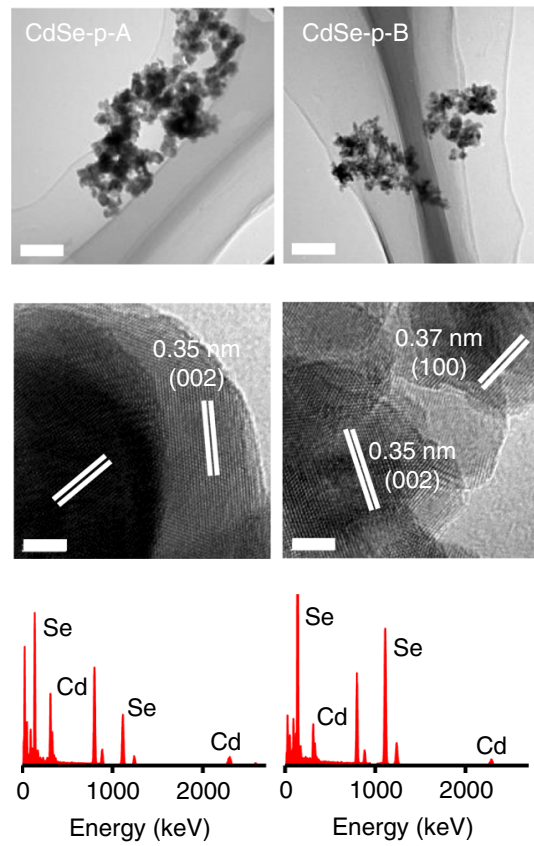

b

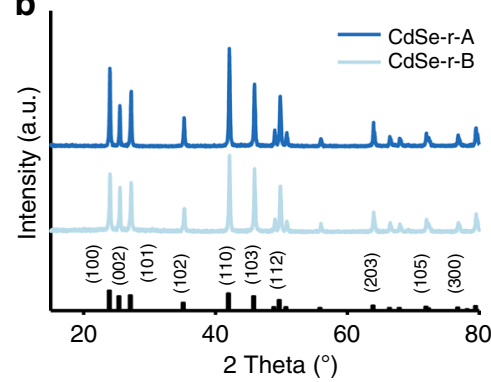

The intensity ratio of $(100) /(002)$

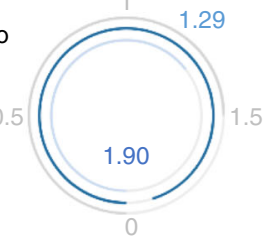

e
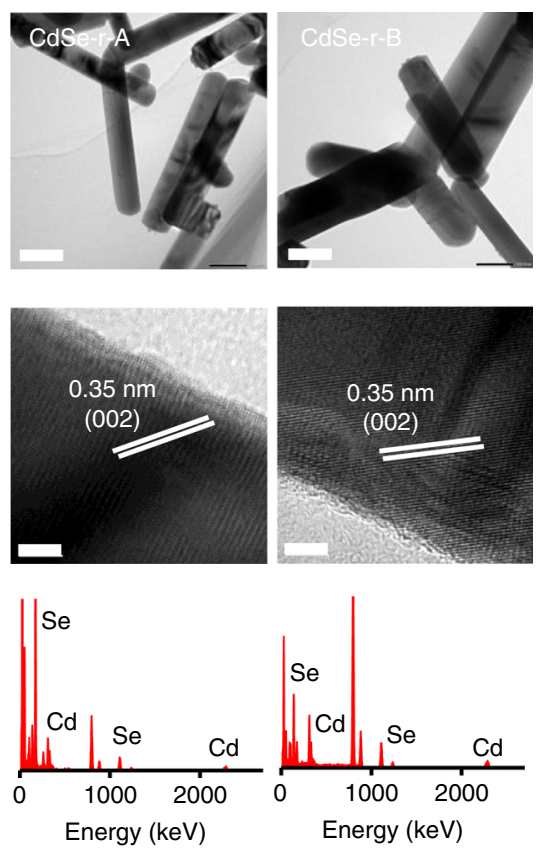
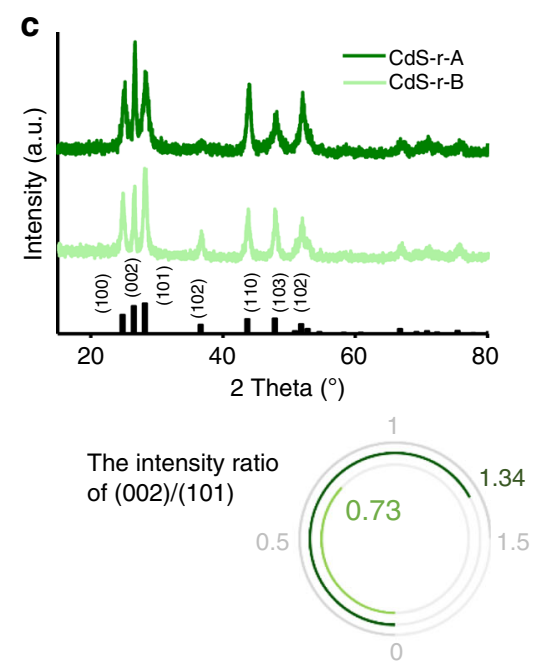

f
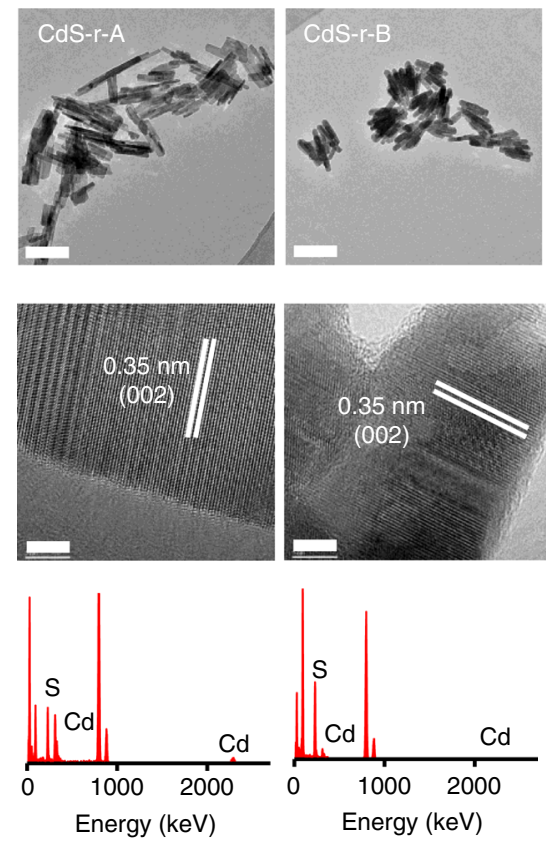

Fig. 1 Characterization of different-faceted CdSe and CdS nanocrystals. a-c X-ray diffraction (XRD) spectra of CdSe nanoparticles (a, CdSe-p-A: dark red solid line; CdSe-p-B: light red solid line), CdSe nanorods (b, CdSe-r-A: dark blue solid line; CdSe-r-B: light blue solid line), and CdS nanorods (c, CdS-r-A: dark green solid line; CdS-r-B: light green solid line). The two nanomaterials in each group had different facet content (i.e., (100) vs. (002) for CdSe and (002) vs. (101) for CdS), according to the intensity ratio of the corresponding peaks in the XRD spectra. d-f Transmission electron microscopy (TEM) images and energy-dispersive spectroscopy (EDX) spectra showed that different-faceted CdSe nanoparticles (d), CdSe nanorods (e), and CdS nanorods (f) exhibited similar size and morphology. TEM analysis was independently repeated five times, and the results were similar. Scale bar of upper panel: $100 \mathrm{~nm}$. Scale bar of middle panel: $5 \mathrm{~nm}$. Source data are provided as a Source Data file.

(Fig. 2), whereas adsorption of the non-thiol transferrin to the Cd nanocrystals appeared to be independent of or less affected by exposed facets and occurred to a much lower extent than with thiol-rich transferrin (Fig. 4c-e).

To further discern the role of thiols in transferrin binding with different facets, competitive adsorption experiments using a model thiol-rich protein (i.e., bovine serum albumin (BSA)) and low-molecular-weight model compounds were conducted (Fig. 4f), and the experimental data were complemented with theoretical computation (Fig. 4g-1). As expected, BSA similarly exhibited preferential binding toward CdSe-p-A relative to CdSe-p-B (Supplementary Fig. 2). Thiol-containing amino acid, cysteine, and its non-thiol analog, serine, were also compared for their adsorption affinities to CdSe-p-A vs. CdSe-p-B. In the same reaction matrix, cysteine outcompeted serine and preferentially bound with nano-CdSe, particularly to a greater extent with the " $A$ " material than the " $B$ " material (Fig. 4f). The specific interaction between thiol and different facets of CdSe were assessed via density functional theory (DFT) calculation, and the favorable adsorption process was indicated by the lower (i.e., more negative) values of the adsorption energy (Fig. 4g-1). All calculated adsorption energies ( -1.62 to $-0.95 \mathrm{eV}$, Fig. $4 \mathrm{i}, 1)$ were sufficiently negative to indicate chemical adsorption pathways. CdSe-(100) facet exhibited lower adsorption energy toward cysteine compared with CdSe-(002) facet, and CdS-(002) facet exhibited lower adsorption energy toward cysteine compared with CdS-(101) facet. Altogether, these results reveal that transferrin associated with $\mathrm{Cd}$ nanocrystals mainly via chemical 
a

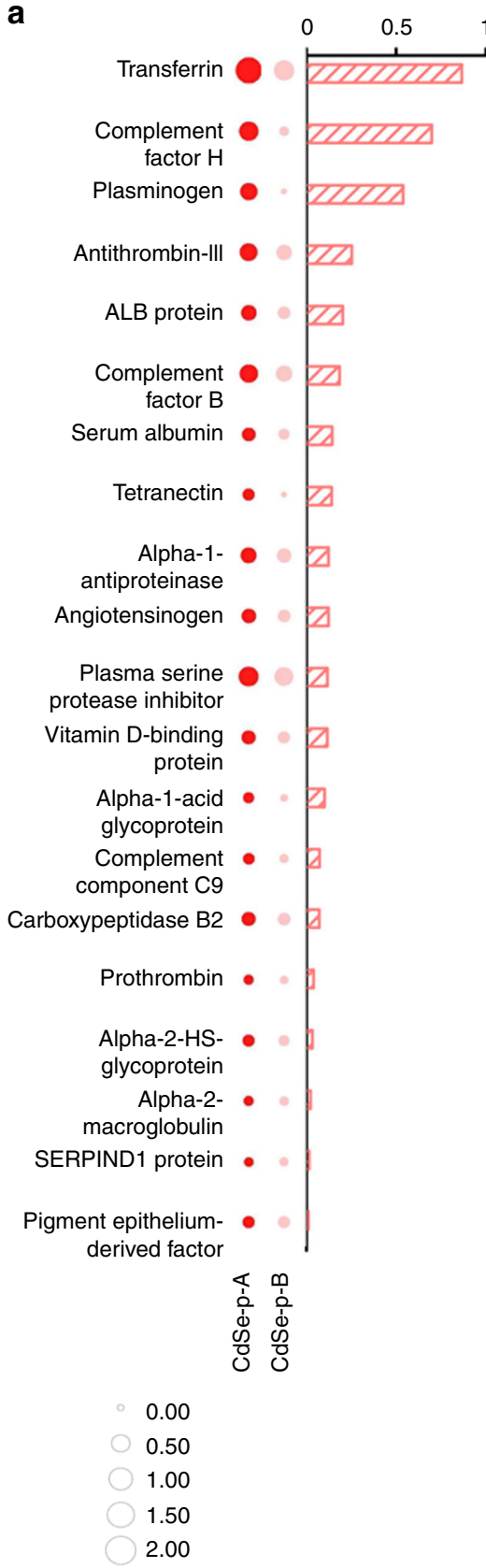

b

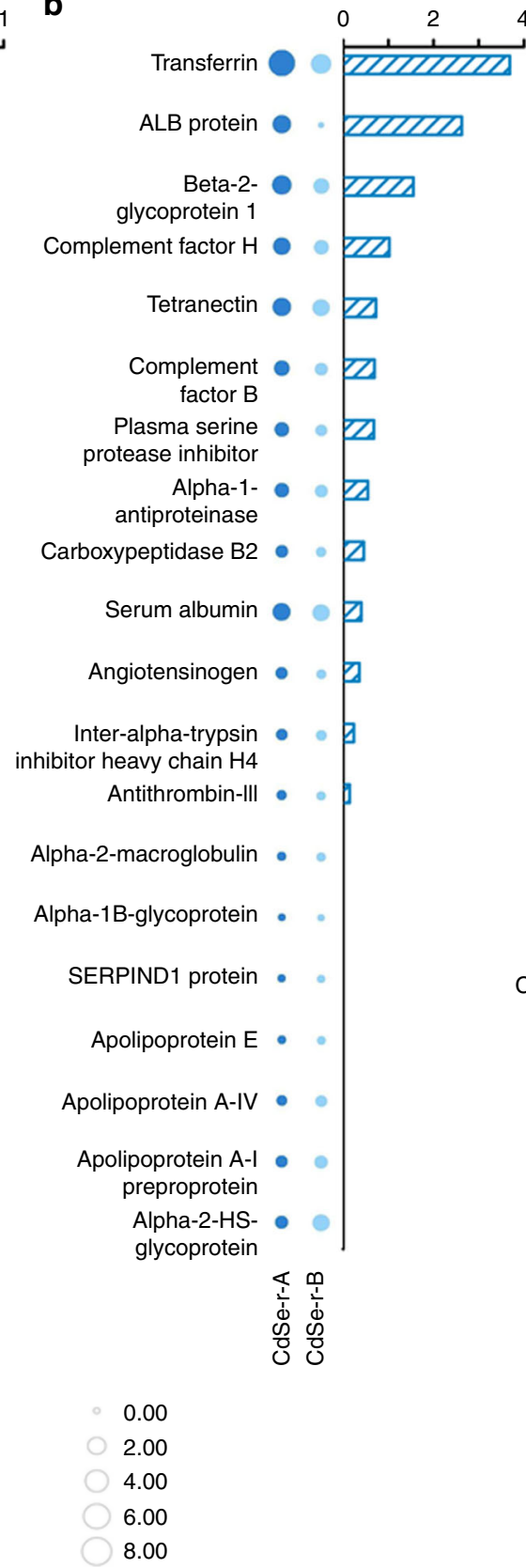

C

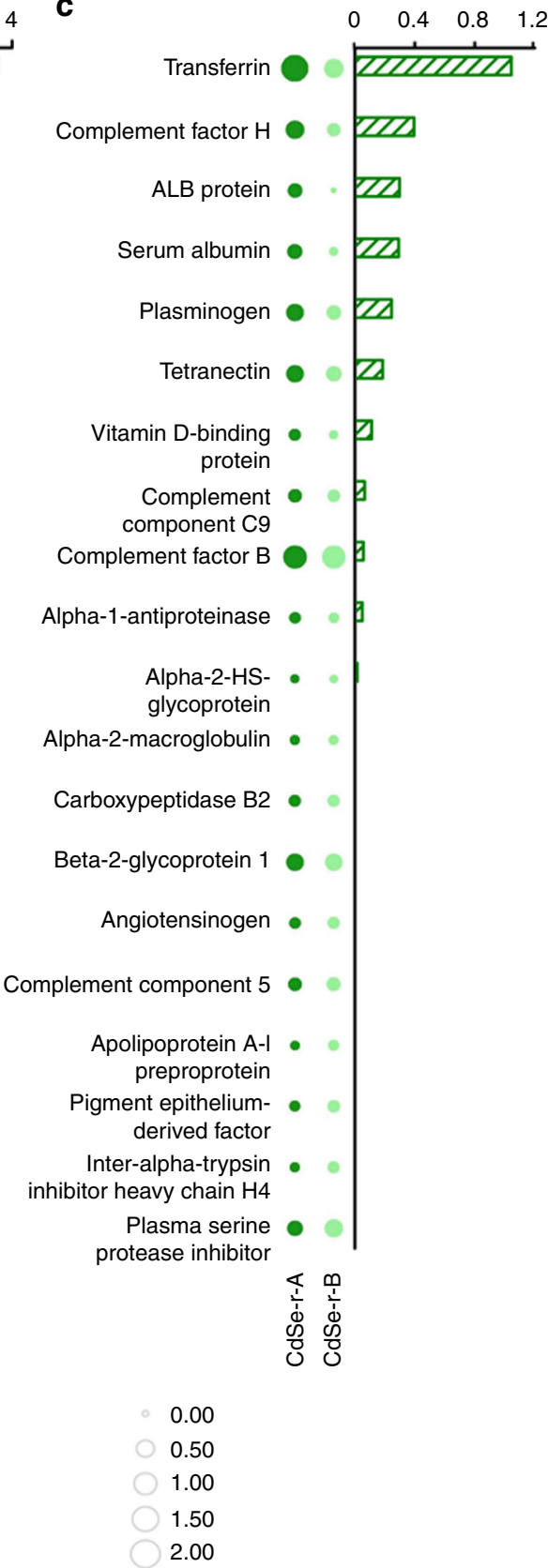

Fig. 2 Transferrin was enriched in the hard protein corona and the extent of enrichment was susceptible to the exposed facets of CdSe and CdS nanocrystals. The protein coronas were formed in fetal bovine serum. The size of the circles represented the enrichment factor of proteins in the hard corona, and the difference in the protein enrichment factors between the two different-faceted materials is shown in the bar graph. The proteins were ranked according to the decreasing difference in the enrichment factor of proteins detected from the hard coronas on the two different-faceted CdSe nanoparticles (a, CdSe-p-A: dark red bubble; CdSe-p-B: light red bubble), CdSe nanorods (b, CdSe-r-A: dark blue bubble; CdSe-r-B: light blue bubble), and CdS nanorods (c, CdS-r-A: dark green bubble; CdS-r-B: light green bubble). Source data are provided as a Source Data file.

adsorption, and this process occurred through inner-sphere thiol complexation and depended on the exposed crystal facet.

Molecular dynamics (MD) simulations were conducted to evaluate the interaction between the entire protein molecules with different crystal facets, to complement the DFT calculation that focused on the thiol moieties. In the MD calculation, we established six initial structures of transferrin for 20-ns simulations by rotating the transferrin $90^{\circ}$ around the $x$ - and $y$-axes (Supplementary Figs. 3 and 4 ), and utilized the most stable structure (i.e., largest contact atom number) for the comparison between (100) and (002) facets of CdSe using 200-ns simulations (Fig. 5a, b). In order to mimic chemical adsorption process, the transferrin molecules were initially placed $2 \mathrm{~nm}$ away from CdSe (100) or (002) surface, and used a spring constant of $1000 \mathrm{~kJ} \mathrm{~mol}^{-1} \mathrm{~nm}^{-2}$ and a pull rate of $0.1 \mathrm{~nm} \mathrm{~ns}^{-1}$ to accelerate the initial adsorption. A harmonic potential was utilized to restrain the $S$ atoms in position after they directly contacted with the CdSe (100) surface 26 . The number of all protein atoms in direct contact with (100) facet gradually increased from 107 to 430 during the simulation (Fig. 5c), reflecting the thermodynamic feasibility of transferrin adsorption to CdSe-(100). In contrast, transferrin molecules were prone to "fleeing away" from the (002) facet, 

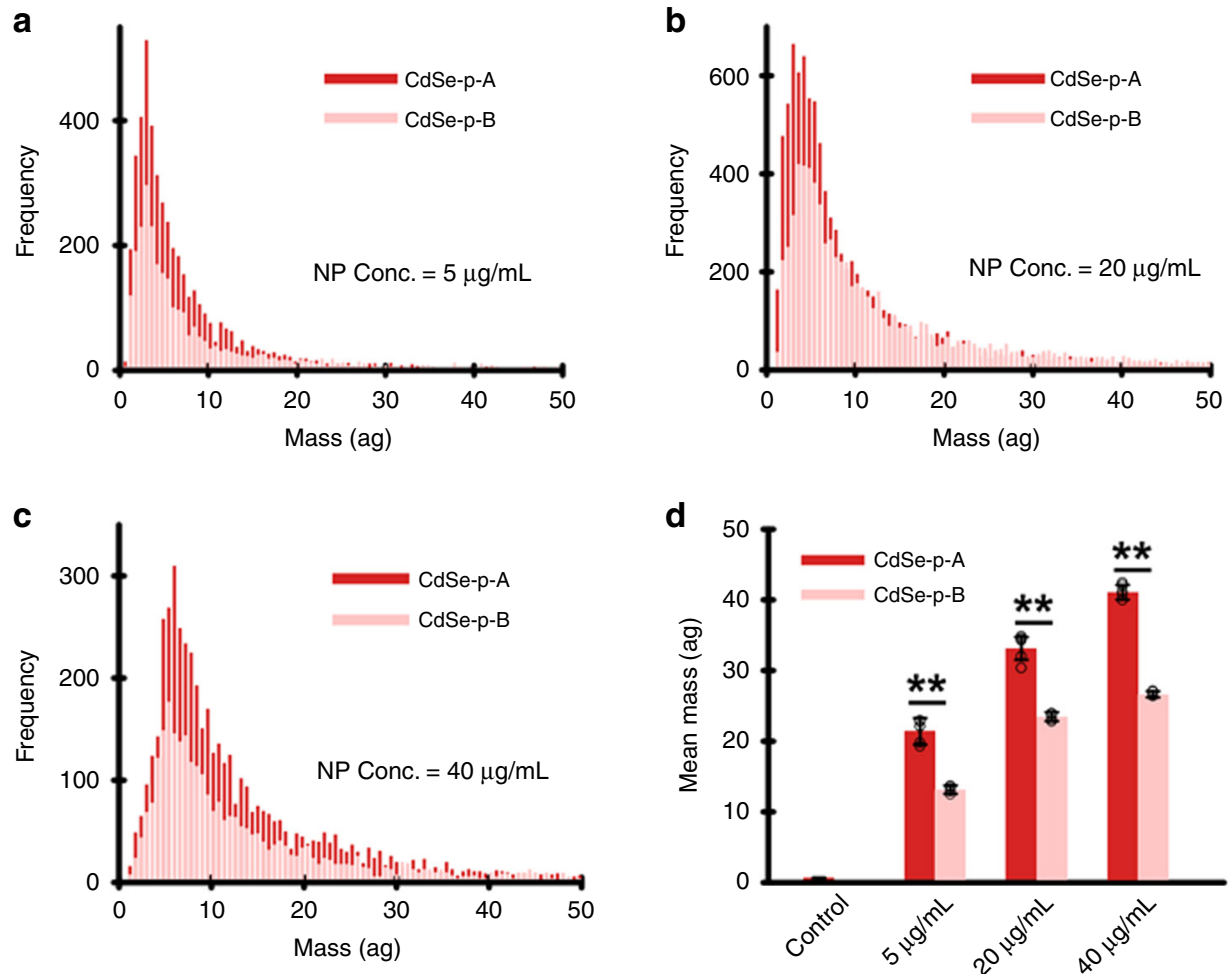

e
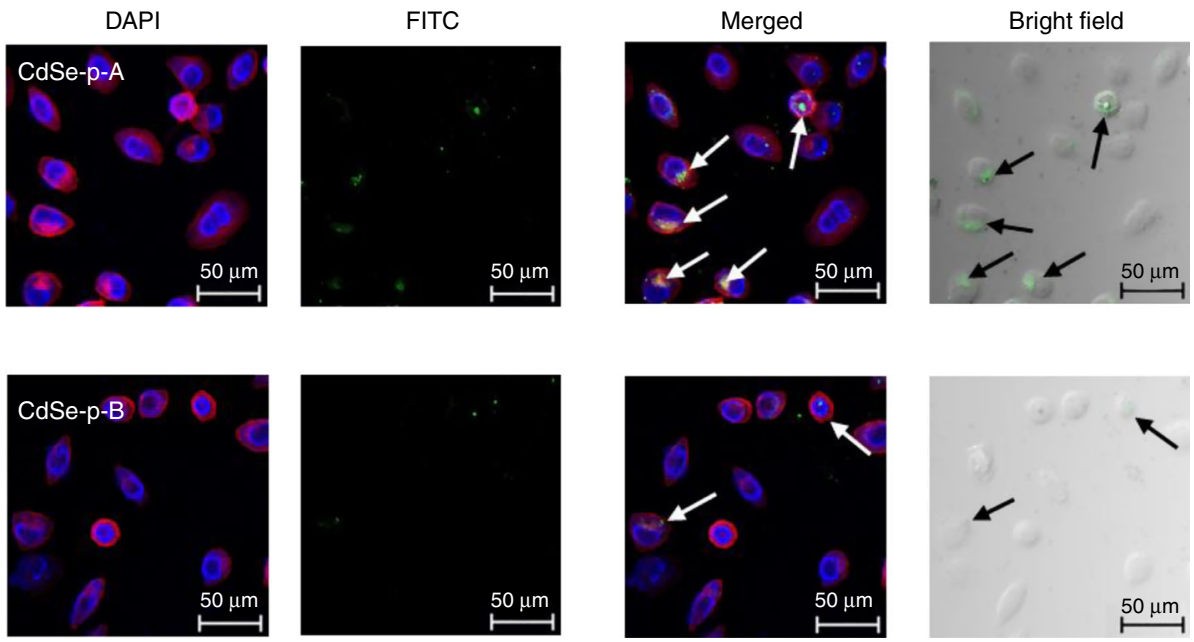

Fig. 3 Facet-dependent binding of transferrin and cellular uptake of CdSe nanoparticles. a-d Single-cell-inductively coupled plasma-mass spectrometry (SC-ICP-MS) histogram of mass distribution (a-c) and mean mass (d) of CdSe nanoparticles (CdSe-p-A: dark red bar; CdSe-p-B: light red bar) taken up by HeLa cells, after cell incubation with transferrin-CdSe conjugates for $3 \mathrm{~h}$. Results show higher cellular content of nano-Cd in HeLa cells exposed to " $A$ " materials (i.e., CdSe with more (100) facet) that preferentially bound with transferrin. Data are presented as mean \pm SD of five replicate samples ( $n=5$; $p=0.0007,0.0004,<0.0001$ for the group of $5,20,40 \mu \mathrm{g} / \mathrm{mL} \mathrm{CdSe}$ nanoparticles by one-way ANOVA, respectively). e Confocal laser scanning microscopy images of HeLa cells after incubated with FITC-labeled transferrin-CdSe conjugates demonstrated greater cellular uptake of "A" materials. Microscopic analysis was independently repeated five times, and the results were similar. Statistical significance between groups: $\left({ }^{\star \star}\right) p<0.01$. Source data are provided as a Source Data file.

indicated by the slight decrease in the contact atom number (Fig. 5c), and thus the transferrin association with the (002) facet was relatively unstable. The sulfur atoms directly contacted with nano-CdSe were all originated from the disulfides moieties of transferrin molecules, with more sulfur binding on (100), relative to (002) (Fig. 5d). There were six sulfur-binding sites, including C118-194, C171-177, and C158-174, on CdSe-(100) facet, as opposed to only four sulfur-binding sites, including C137-331 and C615-620, on CdSe-(002) facet. The smaller fluctuation in the number of contact sulfur atoms on (100) vs. (002), especially toward the end of the simulation (Fig. 5d), suggested that thiol binding on (100) was more stable than that on (002).

Recent studies have reported that water layers adjacent to the material surface play an important role in the adsorption of biomolecules $^{19,29}$. Our MD calculations showed that the difference in the number and arrangement of water molecules in the first solvation shell (FSS) on (100) vs. (002) also contributed to the facet-dependent binding affinity of transferrin. The FSS on the (100) facet contained fewer layers and lower density of water molecules (Fig. 5e, g), compared with the FSS on the (002) facet 
a

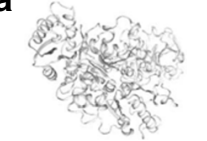

Transferrin (mut)

b

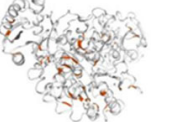

Transferrin

f

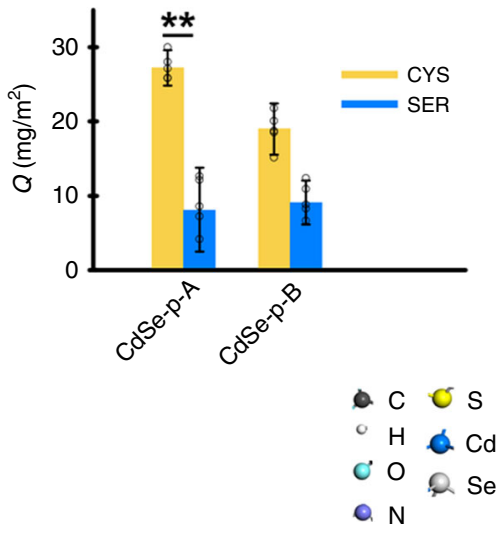

C

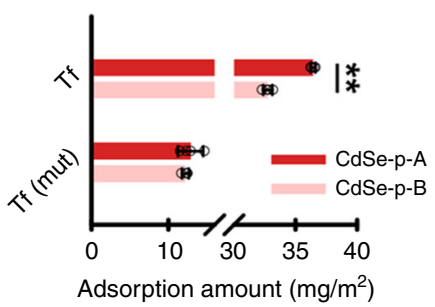

g

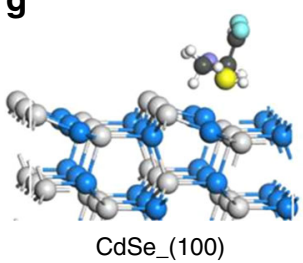

j

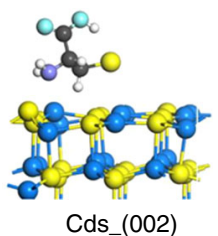

d

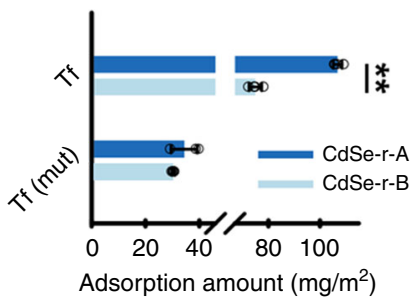

$\mathbf{h}$

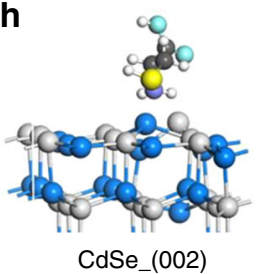

k

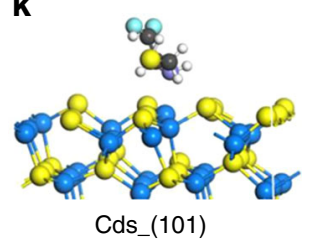

e

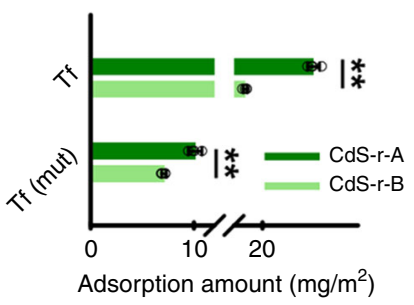

i
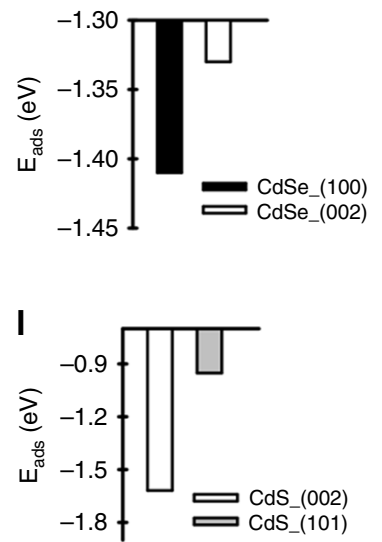

Fig. 4 Facet-dependent preferential binding of transferrin with $\mathbf{C d}$ nanocrystals occurred through thiol complexation. $\mathbf{a}$, $\mathbf{b}$ Schematic description of the structures of transferrin and non-thiol transferrin (mut) (cysteine shown in red). Adsorption of transferrin on CdSe nanoparticles (c, CdSe-p-A: dark red bar; CdSe-p-B: light red bar; $p=0.0006$ for transferrin and 0.4071 for transferrin (mut)), CdSe nanorods (d, CdSe-r-A: dark blue bar; CdSe-r-B: light blue bar; $p<0.0001$ for transferrin and $=0.1306$ for transferrin (mut)) and CdS nanorods (e, CdS-r-A: dark green bar; CdS-r-B: light green bar; $p=0.0003$ for transferrin and 0.0006 for transferrin (mut)) was consistently greater compared with the non-thiol transferrin (mut). Data are presented as mean \pm SD of four replicate samples, and analyzed by one-way ANOVA $(n=4)$. Competitive adsorption of cysteine (CYS) vs. serine (SER) (ff, CYS: yellow bar; SER: blue bar) on the two different-faceted CdSe nanoparticles showed that thiol-containing compounds preferentially adsorbed to the " $A$ " material. Data are presented as mean \pm SD of five replicate samples $(n=5 ; p=0.0098$ for cysteine and 0.8567 for serine by one-way ANOVA). Density function theory (DFT) calculation indicated that cysteine exhibited lower adsorption energy toward (100) vs. (002) of CdSe (g-i, CdSe_(100): black bar; CdSe_(002): white bar), and exhibited lower adsorption energy toward (002) vs. (101) of CdS (j-I, CdS_(002): white bar; CdS_(101): gray bar). Statistical significance between groups: ${ }^{\star \star} p<0.01$. Source data are provided as a Source Data file.

(Fig. 5f, g). The facet-dependent differential affinity to water was corroborated by the adsorption energy values of water molecules on the CdSe-(100) facet $(-0.33 \mathrm{eV})$ vs. CdSe-(002) facet $(-0.66 \mathrm{eV})$ according to the DFT calculation. Due to the relatively low affinity of the (100) facet to water, the hydrogen bonds in the FSS on this facet dominantly formed among water molecules (Fig. 5h). In the FSS on the (002) facet, although the overall abundance of hydrogen bonds was smaller than the (100) facet (Fig. 5i, j), a good fraction of hydrogen bonds formed between water molecules and the (002) facet. As a result, the interfacial water was more tightly bound to the (002) facet than to the (100) facet, which rendered it difficult for transferrin to replace these water molecules prior to forming inner-sphere coordination bonds with the (002) facet. Hence, a less compact and loosely bound water molecule network occurred on the (100) facet and facilitated the approaching process of transferrin toward the facet from bulk solution, which may, at least partly, explain the preferential binding of transferrin to CdSe nanocrystals with larger (100) content (Figs. 2 and 4c-e).

Significant research attention has focused on predicting and manipulating the behavior of engineered nanomaterials in biological environment based on their morphology and surface coating. Our research points to a long underappreciated parameter, the exposed crystal facet. We demonstrate the feasibility of facet modulation for enhancing the receptor-mediated cancer cell delivery of cadmium chalcogenide nanocrystals via preferential and stable chemical adsorption of transferrin to specific exposed facets in a complex biological matrix. This process was controlled by the affinity of the crystal facets for the thiol moieties of proteins as well as for the water molecules in the FSS. Given that chemical complexation is a common mechanism for adsorption of ligand-rich macromolecules onto metal-containing surfaces, the implication of our discovery should not be limited to the case of nano-CdSe and CdS, and may be extended to more biocompatible nanocrystals, particularly those containing soft metals (e.g., $\mathrm{Au}, \mathrm{Ag}, \mathrm{Pt}, \mathrm{Pd}$, and $\mathrm{Zn}$ ). Such nanocrystal-biomolecule complexes should be examined under realistic scenarios (in vivo) prior to considering biomedical applications.

Overall, many thiol-rich proteins besides transferrin (e.g., serum albumin, complement factor $\mathrm{H}$ ) mediate important physiological functions in the human body, and likely modulate biological responses to nanomaterials via specific protein-receptor pathways. Therefore, facet engineering of nanocrystals containing soft metals offers a promising approach for future design of metal-containing nanostructures with improved safety and efficiency in biological applications.

\section{Methods}

Preparation and characterization of nanocrystals. All chemical reagents used were purchased from Sigma-Aldrich (China). The CdSe nanoparticles were synthesized using the following methods ${ }^{30}$. For synthesizing CdSe nanoparticles, $0.048 \mathrm{~g}$ Se powder, $0.133 \mathrm{~g} \mathrm{Cd}(\mathrm{AC})_{2} \cdot 2 \mathrm{H}_{2} \mathrm{O}$, and $0.2 \mathrm{~g} \mathrm{NaOH}$ were mixed in $20 \mathrm{~mL} n$-butyl 


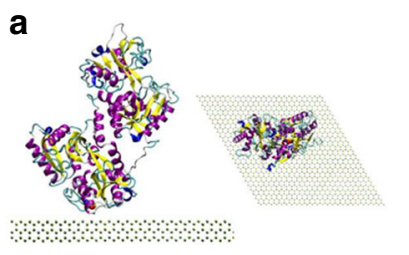

$\mathrm{CdSe}_{-}(100)$

e



h

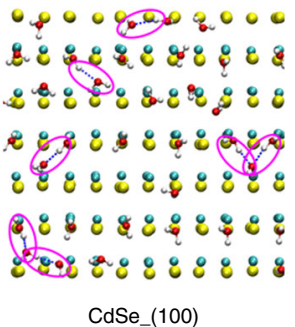

b

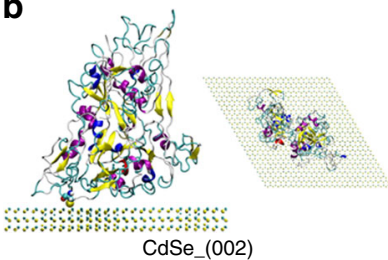

f

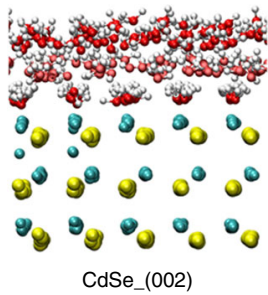

i

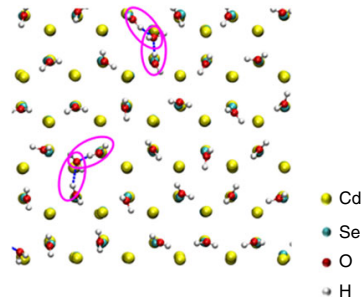

C

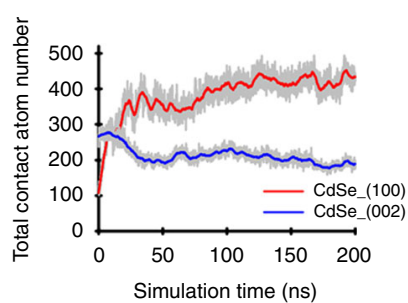

d

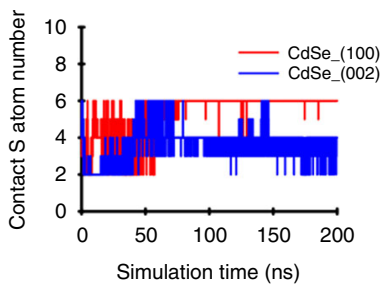

g

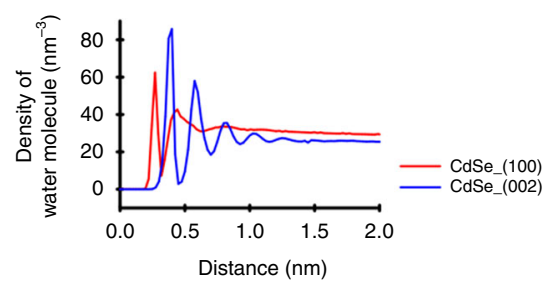

j

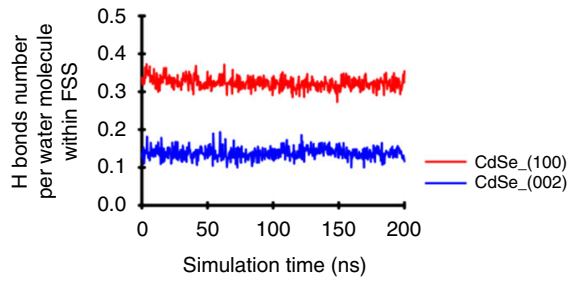

Fig. 5 Molecular dynamics (MD) simulation of interaction between transferrin with CdSe (100) and (002) facets. Conformation of transferrin was different on (100) facet (a) and (002) facet (b), which resulted in different total contact atom number (c, CdSe_(100): red solid line; CdSe_(002): blue solid line) and contact sulfur atom number (d, CdSe_(100): red solid line; CdSe_(002): blue solid line). Snapshot of water molecule layers (e, f), number density of water molecules along the $z$-direction ( $\mathbf{g}$, CdSe_(100): red solid line; CdSe_(002): blue solid line), and the network (h, $\mathbf{i})$ and number (j, CdSe_ (100): red solid line; CdSe_(002): blue solid line) of hydrogen bonds in the first solvation shell of CdSe (100) and (002) facets. The hydrogen bonds were shown as blue dash lines and highlighted in purple circles $(\mathbf{h}, \mathbf{i})$. Source data are provided as a Source Data file.

alcohol, and heated at $150^{\circ} \mathrm{C}$ for $11 \mathrm{~h} .0 .2 \mathrm{~g}$ EDTA was added during synthesis of CdSe-p-B nanomaterials. For synthesizing CdSe nanorod A (CdSe-r-A), $3.08 \mathrm{~g} \mathrm{Cd}$ $\left(\mathrm{NO}_{3}\right)_{2} \cdot 4 \mathrm{H}_{2} \mathrm{O}$ was dissolved in $10 \mathrm{ml}$ of DI water, and then $\mathrm{NH}_{3} \cdot \mathrm{H}_{2} \mathrm{O}$ was slowly added into the solution to adjust $\mathrm{pH}$. In another solution, $0.86 \mathrm{~g} \mathrm{Na}_{2} \mathrm{SeO}_{3}$ was stirred for 5 min with $15 \mathrm{ml}$ of $\mathrm{N}_{2} \mathrm{H}_{4} \cdot \mathrm{H}_{2} \mathrm{O}$ and then was mixed with the $\mathrm{Cd}$ solution. The $\mathrm{pH}$ of the solution was adjusted to 11 and heated at $180^{\circ} \mathrm{C}$ for $4 \mathrm{~h}^{31}$. For CdSe-r-B, $0.039 \mathrm{~g}$ Se powder was mixed with hydroxides $(\mathrm{NaOH}$ : $\mathrm{KOH}=51.5$ : 48.5), $2 \mathrm{~mL} \mathrm{~N}_{2} \mathrm{H}_{4} \cdot \mathrm{H}_{2} \mathrm{O}$, and $8 \mathrm{~mL}$ DI water. Then $0.154 \mathrm{~g} \mathrm{Cd}\left(\mathrm{NO}_{3}\right)_{2} \cdot 4 \mathrm{H}_{2} \mathrm{O}$ was added, and the solution was heated at $200{ }^{\circ} \mathrm{C}$ for $24 \mathrm{~h}^{32}$. For synthesizing CdS nanorod A (CdS-r-A), $0.3 \mathrm{~g}$ of $\mathrm{CdCl}_{2}$ and $0.4 \mathrm{~g}$ of thiourea were mixed with $2 \mathrm{~mL}$ of DI water and $18 \mathrm{~mL}$ of ethylenediamine. Then the solution was heated at $150^{\circ} \mathrm{C}$ for $5 \mathrm{~h}^{23}$. For CdS-r- $\mathrm{B}, 0.266 \mathrm{~g} \mathrm{Cd}(\mathrm{AC})_{2} \cdot 2 \mathrm{H}_{2} \mathrm{O}$ and $0.079 \mathrm{~g} \mathrm{~L}$-cysteine were dissolved in $2 \mathrm{ml}$ DI water. Then, $18 \mathrm{~mL}$ ethanolamine (EA) was added to the solution, which was then heated at $180^{\circ} \mathrm{C}$ for $24 \mathrm{~h}^{33}$.

X-ray diffraction (XRD) spectra of the synthesized CdSe and CdS nanomaterials were obtained using Rigaku D/Max III diffractometer (D/MAX2500, Rigaku Inc., Japan) with $\mathrm{Cu} \mathrm{K}$ radiation, $\lambda=1.5418 \AA$ A. Physical dimensions and morphologies of the materials were characterized by transmission electron microscopy (TEM) coupled with energy-dispersive spectroscopy (EDX) (JEM-2100, JEOL, Japan). The $D_{\mathrm{h}}$ and $\xi$-potential of the CdSe and CdS nanomaterials were determined using a ZetaSizer (Nano ZS, Malvern Instruments, UK). The Brunauer-Emmett-Teller (BET)-specific surface area was determined by multipoint $\mathrm{N}_{2}$ adsorptiondesorption method using an accelerated surface area and porosimetry system (ASAP2010, Micromeritics Co., USA).

Characterization of protein corona on nanocrystals. The CdSe and CdS suspension was sonicated $(100 \mathrm{~W})$ for $30 \mathrm{~min}$ before incubating with $10 \%(\mathrm{v} / \mathrm{v})$ fetal bovine serum (FBS, Genview, China) at $37^{\circ} \mathrm{C}$. After $4 \mathrm{~h}$, the CdSe/CdS-protein complexes were separated from the supernatant plasma by centrifugation at $12,396 \times g\left(4^{\circ} \mathrm{C}\right)$ for $10 \mathrm{~min}$. The pellet was washed with phosphate buffer saline (PBS) three times to remove the proteins with low affinity for the surface of $\mathrm{CdSe} / \mathrm{CdS}$ nanomaterials (i.e., the soft protein corona).

The composition of the hard protein corona formed on the surface of nanoCdSe and CdS were characterized using liquid chromatography-mass spectrometry/mass spectrometry (LC-MS/MS). All chemical reagents used here were purchased from Sigma-Aldrich (China). For LC-MS/MS analysis, proteins were digested in solution containing $20 \mu \mathrm{L}$ trypsin overnight at $37^{\circ} \mathrm{C}$. The enzymatic hydrolysis was terminated by the addition of $0.1 \%$ formic acid. After digestion, the peptide mixtures were analyzed by LC-MS/MS using an ultimate 3000 nanoLC system (Dionex Inc., USA) and a tandem mass spectrometry (MS/ MS) in a Q Exactive (Thermo scientific Inc., USA). Peptides were separated using a $\mathrm{C}_{18}$ trap column (Thermo scientific Inc., USA) with DI water $\left(\mathrm{A}: \mathrm{H}_{2} \mathrm{O}, 0.1 \% \mathrm{FA}\right)$ and acetonitrile (B: ACN, $0.1 \% \mathrm{FA}$ ) as eluents. The MS was operated in the positive ion mode and the $\mathrm{m} / \mathrm{z}$ ratio of $350-2000$ was scanned. For protein identification, raw data files were converted to Mascot generic format (mgf) files and searched in National Center for Biotechnology Information (NCBInr) database via Mascot Search (version 2.3.01, Matrix Science, http://www.matrixscience.com/). Mascot searching parameters included trypsin as the proteolytic enzyme with one missed cleavage. The modifications of methionine oxidation were selected. Peptide charge was set to $+1,+2$, and +3 . A minimal Mascot score of 100 was set for protein identity validation. The 20 most abundant proteins (according to exponentially modified protein abundance index, emPAI values) in hard corona of each material were chosen for comparison between different-faceted materials. The enrichment factor of protein fractions were the emPAI ratio of the protein fractions (normalized with respect to BET surface area) associated with the nanocrystals with respect to the fractions in $\mathrm{FBS}^{24}$.

Cell silencing and cellular uptake of nano-CdSe. HeLa cells were seeded on tissue-culture-treated six-well plates at a density of 300,000 cells per well and incubated at $37^{\circ} \mathrm{C}\left(5 \% \mathrm{CO}_{2}\right)$ for $24 \mathrm{~h}$ to ensure adhesion onto the tissue-culture plate surface. Briefly, 100 pmol small interfering RNA (siRNA) oligo (GenePharma Biotechnology, China) for each well were transfected into cells using Lipofectamine 2000 (Invitrogen) according to the manufacturer's instructions. Two siRNA sequences (siRNA-2423 and siRNA-2061, respectively) were synthesized as follows: for siRNA-2423, sense strand was $5^{\prime}$-GAACUUGAAACUGCGUAAATT- 3 ' and antisense strand was 5'-UUUACGCAGUUUCAAGUUCTT-3'; for siRNA-2061, sense strand was $5^{\prime}$-GCUGGUCAGUUCGUGAUUATT- $3^{\prime}$ and antisense strand was 5'-UAAUCACGAACUGACCAGCTT-3'. The silencing effects were confirmed by western blot experiments (Supplementary Fig. 1). Cells were transfected with siRNAs for $48 \mathrm{~h}$ in all experiments before exposure to CdSe nanoparticles. After silencing, cells were washed by DMEM medium and then replaced by nanoparticle dispersions (i.e., diluted by DMEM with $10 \%$ FBS to concentration of 
$40 \mu \mathrm{gL}^{-1}$ ) and incubated for $3 \mathrm{~h}$ before harvesting. The samples were digested by dry baths/block heaters (Thermo Scientific, China), and the cellular content of Cd was quantified using an inductively coupled plasma-mass spectrometer (ICP-MS, Agilent 8800, Agilent Technologies, Inc., China).

Cellular uptake of nano-CdSe analyzed by SC-ICP-MS. HeLa cells were cultured in Dulbecco's Modified Eagle's medium (DMEM, Gibco BRL Life Technologies Inc., USA) supplemented with 10\% FBS (Gibco BRL Life Technologies Inc., USA) and 100 units $\mathrm{mL}^{-1}$ penicillin/streptomycin (Invitrogen, USA) in a humidified $5 \% \mathrm{CO}_{2}$-balanced air incubator at $37^{\circ} \mathrm{C}$. For the CdSe nanoparticle uptake experiment, HeLa cells were seeded on tissue-culture-treated 12 -well plates at a density of 50,000 cells per well and incubated at $37^{\circ} \mathrm{C}\left(5 \% \mathrm{CO}_{2}\right)$ for $24 \mathrm{~h}$ to ensure adhesion onto the tissue-culture plate surface. The cells were then exposed to CdSe nanoparticles at a concentration of 5, 20, and $40 \mu \mathrm{g} \mathrm{mL}^{-1}$ in DMEM (supplemented with $10 \%$ FBS) for $3 \mathrm{~h}$. Next, the cells were washed with PBS five times, enzymatically detached from the tissue-culture plate surface, and fixed in PBS to a final concentration of $\sim 100,000$ cells $\mathrm{mL}^{-1}$.

All analysis were done on a NexION 2000 inductively coupled plasma-mass spectrometer (ICP-MS) with the Single Cell Application Module in Syngistix software (v 4.0) using the conditions in Supplementary Table 2. The components used for SC-ICP-MS analysis included the NexION 2000 Asperon $^{\text {tox }}$ spray chamber as well as the specialized Single Cell Micro DX Autosampler. The Asperon spray chamber was designed to maximize cell transport to the NexION, while the Single Cell Micro DX Autosampler agitated the cellular suspensions prior to analysis to ensure that the cells did not settle from the suspension.

Cellular uptake of nano-CdSe analyzed by confocal microscopy. CdSe nanoparticles were incubated with FITC-labeled transferrin (Thermo Fisher Scientific, USA) to form CdSe-transferrin conjugates in PBS. Afterward, HeLa cells were exposed to $20 \mu \mathrm{g} \mathrm{mL}{ }^{-1} \mathrm{CdSe}$-transferrin conjugates for $3 \mathrm{~h}$, washed with PBS three times, fixed with $3.7 \%$ paraformaldehyde for $10 \mathrm{~min}$, and then incubated with $0.1 \%$ Triton X-100 for $5 \mathrm{~min}$. The cell plasma membranes were stained with rhodamine phalloidin (Solarbio Inc., China), and the cell nuclei were stained with 4',6diamidino-2-phenylindole (DAPI, Beyotime, China). Fluorescence images were captured using a TCS SP8 laser scanning confocal microscope (Leica, Germany).

Expression and purification of non-thiol transferrin mutant. For the expression of the transferrin (mut) proteins, key operational parameters, including sequencing information, are include in Supplementary Table 3. The non-thiol transferrin mutant was synthesized by and available from Beijing Protein Innovation, China. Competent cells of Escherichia coli. (BL21) kept at $-80^{\circ} \mathrm{C}$ were slowly thawed on ice and then incubated with plasmids (pET30a) containing modified genetic sequences (i.e., with cysteines replaced by glycines) and tag (HIS) on ice for $30 \mathrm{~min}$, heat shocked at $42^{\circ} \mathrm{C}$ for $90 \mathrm{~s}$, and cooled on ice for $2 \mathrm{~min}$. In total, $800 \mu \mathrm{L}$ kanamycin-free LB medium was added to the cells, and the cell suspension was incubated at $37^{\circ} \mathrm{C}$ for $45 \mathrm{~min}$. After centrifugation at $2152 \times \mathrm{g}$ for $3 \mathrm{~min}$, the pellets were collected and inoculated onto agar plates containing LB medium with Kanamycin. The plates were dried and incubated at $37^{\circ} \mathrm{C}$ overnight.

The colonies were transferred into fresh LB medium with kanamycin and kept at $37^{\circ} \mathrm{C}$ on a shaker $(200 \mathrm{rpm})$ until $\mathrm{OD}_{550}$ reached $0.6-0.8$. Then the cultures were incubated with $0.5 \mathrm{mM}$ of isopropyl $\beta$-D-thiogalactoside (IPTG) at $37^{\circ} \mathrm{C}$ for another $2 \mathrm{~h}(200 \mathrm{rpm})$. After incubation, the colonies were extracted by centrifugation for $1 \mathrm{~min}(12,396 \times g)$, and then lysed by lysis buffer $(10 \mathrm{mM}$ Tris$\mathrm{HCl}, \mathrm{pH}$ 8.0). The samples were then boiled at $100^{\circ} \mathrm{C}$ for $5 \mathrm{~min}$ with loading buffer and analyzed by SDS-PAGE to confirm protein expression.

Next, the colonies were transferred into fresh LB medium with kanamycin and kept at $37^{\circ} \mathrm{C}$ on a shaker $(200 \mathrm{rpm})$ until $\mathrm{OD}_{550}$ reached $0.6-0.8$. Then the cultures were incubated with $0.5 \mathrm{mM}$ of IPTG at $16^{\circ} \mathrm{C}$ overnight. After incubation, the cell cultures were centrifuged at $5510 \times g$ for $6 \mathrm{~min}$, and the pellets were collected and resuspended in $10 \mathrm{mM}$ Tris- $\mathrm{HCl}$ ( $\mathrm{pH} 8.0)$ prior to ultrasonic disruption $(500 \mathrm{~W}$, 5 -s intervals, 180 cycles). Then, $100 \mu$ l of processed culture was centrifuged at $12,396 \times g$ for $10 \mathrm{~min}$, and the pelleted proteins were resuspended in $50 \mu \mathrm{L} 10 \mathrm{mM}$ Tris- $\mathrm{HCl}(\mathrm{pH}$ 8.0) prior to SDS-PAGE analysis, which found that the target proteins were in the culture pellet instead of supernatant.

To purify target proteins, a nickel-affinity column (Ni-Sepharose 6 Fast Flow, GE Healthcare, USA) with immobilized nickel ions was applied after equilibrated with a loading buffer containing $10 \mathrm{mM}$ Tris- $\mathrm{HCl}$ (pH 8.0), $8 \mathrm{M}$ urea, and $0.5 \mathrm{M}$ $\mathrm{NaCl}$. The target proteins were intercepted by the column and then eluted with imidazole at gradually increasing concentrations of $15,60,500 \mathrm{mM}$ in $10 \mathrm{mM}$ Tris$\mathrm{HCl}(\mathrm{pH}$ 8.0), and the purified proteins were collected from eluent. The purity of transferrin (mut) was assessed using SDS-PAGE analysis. After elution, the sample was dialyzed with buffer ( $1 \%$ glycine, $0.1 \%$ SDS, $5 \%$ glycerine, and $10 \mathrm{mM}$ Tris$\mathrm{HCl}, \mathrm{pH} 8.0)$ containing gradient concentration $(6 \mathrm{M}, 4 \mathrm{M}$ and $2 \mathrm{M}$, each concentration for $3 \mathrm{~h}$ ) of urea at $4{ }^{\circ} \mathrm{C}$. Then the sample was dialyzed with buffer containing $1 \%$ glycine, $0.1 \%$ SDS, $5 \%$ glycerine, and $10 \mathrm{mM}$ Tris- $\mathrm{HCl}(\mathrm{pH} 8.0)$ for another $3 \mathrm{~h}$. After dialyzed with $10 \mathrm{mM}$ Tris- $\mathrm{HCl}(\mathrm{pH} 8.0)$ for $3 \mathrm{~h}$, the sample was centrifuged at $12,396 \times g$ for $10 \mathrm{~min}$, and the target proteins were collected in the supernatant.
Adsorption experiments using model biomolecules. For adsorption experiments with low-molecular-weight model compounds, a series of 20-ml vials containing $800 \mu \mathrm{L}$ of $5 \mathrm{mg} \mathrm{mL}^{-1}$ CdSe stock suspension were prepared. Then, amino acids stock solution (in PBS) was added to the vials, and the total volume was adjusted to $10 \mathrm{ml}$ with PBS. The CdSe nanoparticle concentration in these vials was $400 \mu \mathrm{g} \mathrm{mL}^{-1}$ and amino acid concentration was $1000 \mu \mathrm{g} \mathrm{L}^{-1}$, respectively. The vials were shaken at $150 \mathrm{rpm}$ for $4 \mathrm{~h}$ to reach adsorption equilibrium. Afterward, the complexes were separated by centrifugation at $12,396 \times g, 4^{\circ} \mathrm{C}$ for $10 \mathrm{~min}$, and the supernatant was withdrawn to analyze the concentrations of amino acid. The concentrations of cysteine and serine were determined according to the following protocol ${ }^{34}$. Sample aliquots were derivatized by 2,2'-dithiobis(5-nitropyridine) (DTNP, Sigma-Aldrich, China), and cysteine concentration was quantified using a microplate reader (Synergy H4, Bio tek, USA). The total concentration of amino acids was determined using the Total Amino Acid Assay Kit (Nanjing Jiancheng Bioengineering Institute, China), following the manufacturer's instructions. The concentration of serine was calculated based on mass balance.

For adsorption of model proteins (i.e., transferrin, transferrin (mut) and BSA) on $\mathrm{CdSe} / \mathrm{CdS}$ nanomaterials, same procedures were conducted except that the supernatant was withdrawn to analyze the concentrations of proteins using the Bradford Protein Assay Kit (Sangon Biotech, China), following the manufacturer's instructions. For transferrin adsorption, the concentration of transferrin was $50 \mu \mathrm{g} \mathrm{mL}^{-1}$, and the concentration of CdSe nanoparticles was $100 \mu \mathrm{g} \mathrm{mL}^{-1}$. For BSA adsorption, the concentration of BSA was $100 \mu \mathrm{g} \mathrm{mL}^{-1}$, and the concentration of CdSe nanoparticles was 200,400 , and $800 \mu \mathrm{g} \mathrm{mL}{ }^{-1}$, respectively. The blank (i.e., samples without $\mathrm{CdSe}$ ) showed no adsorption of proteins to the vials, and $\mathrm{pH}$ remained constant during the time course of all experiments. The adsorbed mass at each equilibrium concentration was calculated based on a mass balance approach.

Density functional theory (DFT) computational methods. All DFT calculations were implemented using the Vienna Ab-initio Simulation Package (VASP). The electron-ion interaction was described by projector-augmented wave method with a plane-wave cutoff of $420 \mathrm{eV}$. The exchange and correlation potential were described by Perdew-Burke-Ernzerh (PBE) method ${ }^{35-37}$. The van der Waals force was described by DFT-D2 method of Grimme ${ }^{38}$. Spin polarization was considered in all computations. The electronic structure calculations were employed with a Gaussian smearing of $0.1 \mathrm{eV}$. For adsorption energy to cysteine on CdSe/CdS surface, the Brillouin zone was sampled with $3 \times 3 \times 1$ Monkhorst-Pack k-point grids, and for adsorption energy to $\mathrm{H}_{2} \mathrm{O}$, the Brillouin zone was sampled with $5 \times$ $5 \times 4,2 \times 2 \times 1$ and $2 \times 1 \times 1$, Monkhorst-Pack grids, respectively, for CdSe bulk, and CdSe (002), CdSe (100) surfaces calculations ${ }^{39}$. The optimized lattice parameters of wurtzite CdSe were $a=4.20, b=4.20, c=6.84$. A $(3 \times 2)$ surface unit cell with a four-layer slab for the $(100)$ surface and a $(3 \times 3)$ surface unit cell with a four-layer slab for the (002) surface were used. All slabs were spaced more than $14 \AA$ perpendicular to the slab surface to avoid artificial interaction due to periodicity. During optimization, the atoms of two layers at the bottom were fixed, and the remaining atoms were relaxed to reach stable configurations. Atoms were optimized until the residual forces were below $0.02 \mathrm{eV}^{-1}$. The adsorption energies were calculated by the Eq. (1):

$$
E_{\text {ads }} A=E_{\text {slab }+A}-E_{\text {slab }}-E_{A}
$$

Molecular dynamics (MD) simulations. The coordinate of transferrin crystal structure and crystal structure of CdSe was obtained from RCSB Protein Data Bank (PDB entry: $3 \mathrm{~V} 83)^{40}$, Materials Project ${ }^{41}$, and other references ${ }^{42}$, respectively. Six initial structures of transferrin for simulations were obtained by rotating the transferrin $90^{\circ}$ around $x$ - and $y$-axes. Molecular dynamics simulations were performed with GROMACS 5.0.4 package ${ }^{43-45}$ with Amber 99SB-ILDN all-atoms force field ${ }^{46}$ in the NPT ensemble. The sulfur atoms in cysteine of transferrin were all in disulfide bonds form. In order to accelerate the binding process of transferrin a pulling force with a constant pulling speed $1 \mathrm{~nm} \mathrm{~ns}^{-1}$ along the $z$-direction was applied on the sulfur atoms in disulfide bond close to the CdSe surface. To mimic the chemical adsorption of sulfur atoms on CdSe surface, the disulfide bond was constrained to their position on $z$-direction once the sulfur atoms were in direct contact with CdSe surface (with a distance smaller than $0.35 \mathrm{~nm}$ ). For each proteinmaterial simulation group, $20 \mathrm{~ns}$ MD simulation was applied, and then two represented (most stable) groups were chosen for longer simulation time (i.e., $200 \mathrm{~ns}$ ). Temperature was maintained at $300 \mathrm{~K}$ by applying the Nose-Hoover thermostat coupling ${ }^{47}$. The cutoff switching function for non-bonded van der Waals interaction started at $1.2 \mathrm{~nm}$ and reached zero at $1.35 \mathrm{~nm}$. Particle mesh Ewald $^{48}$ summation was used to calculate the long-range electrostatic interactions with a cutoff distance of $1.2 \mathrm{~nm}$ for the separation of the direct and reciprocal space. The bond lengths were constrained by linear constraint solver algorithm ${ }^{49}$, and periodic boundary conditions were applied in all simulations. Protein and materials were dissolved in simple point charge water molecules ${ }^{50}$. The system was neutralized by sodium and chloride ions. Simulations were carried out with a time step of $2 \mathrm{fs}$, and data were saved every 4 ps. The free VMD software (1.9.2) was used to visualize simulation results ${ }^{51}$

Statistical analysis. All data were represented as the mean \pm standard deviation (SD). Statistical analysis was performed using independent one-way ANOVA with 
Statistical Product and Service Solutions (SPSS, 20.0). P-value less than $0.01(p<$ 0.01 ) was considered statistically significant.

Reporting summary. Further information on research design is available in the Nature Research Reporting Summary linked to this article.

\section{Data availability}

The data that support the findings of this study are available from the authors on reasonable request. The coordinate of transferrin crystal structure was obtained from RCSB Protein Data Bank (PDB entry: 3V83, https://www.rcsb.org/structure/3V83). The source data underlying Figs. 1a-c, 2a-c, 3a-d, 4c-f, i-l, 4c, d, g, j and Supplementary Figs. 1-4 and Table 1 are provided as a Source Data file.

Received: 26 November 2019; Accepted: 12 February 2020;

Published online: 09 March 2020

\section{References}

1. Chan, W. C. W. \& Nie, S. Quantum dot bioconjugates for ultrasensitive nonisotopic detection. Science 281, 2016-2018 (1998).

2. Maxwell, D. J., Taylor, J. R. \& Nie, S. Self-assembled nanoparticle probes for recognition and detection of biomolecules. J. Am. Chem. Soc. 124, 9606-9612 (2002).

3. Gill, R., Zayats, M. \& Willner, I. Semiconductor quantum dots for bioanalysis. Angew. Chem. Int. Ed. 47, 7602-7625 (2008).

4. Nykypanchuk, D., Maye, M. M., Lelie, Dvd \& Gang, O. DNA-guided crystallization of colloidal nanoparticles. Nature 451, 549-552 (2008).

5. Mahon, E., Salvati, A., Bombelli, F. B., Lynch, I. \& Dawson, K. A. Designing the nanoparticle-biomolecule interface for "targeting and therapeutic delivery". J. Control Release 161, 164-174 (2012).

6. Boisselier, E. \& Astruc, D. Gold nanoparticles in nanomedicine: preparations, imaging, diagnostics, therapies and toxicity. Chem. Soc. Rev. 38, 1759-1782 (2009).

7. Singh, R. \& Lillard, J. W. Jr. Nanoparticle-based targeted drug delivery. Exp. Mol. Pathol. 86, 215-223 (2009).

8. Yang, Y. et al. Aptazyme-gold nanoparticle sensor for amplified molecular probing in living cells. Anal. Chem. 88, 5981-5987 (2016).

9. Nel, A. E. et al. Understanding biophysicochemical interactions at the nanobio interface. Nat. Mater. 8, 543-557 (2009).

10. Sperling, R. A. \& Parak, W. J. Surface modification, functionalization and bioconjugation of colloidal inorganic nanoparticles. Philos. Trans. R. Soc. A 368, 1333-1383 (2010)

11. Salvati, A. et al. Transferrin-functionalized nanoparticles lose their targeting capabilities when a biomolecule corona adsorbs on the surface. Nat Nanotechnol. 8, 137-143 (2013).

12. Herda, L. M., Hristov, D. R., Giudice, M. C. L., Polo, E. \& Dawson, K. A Mapping of molecular structure of the nanoscale surface in bionanoparticles. J. Am. Chem. Soc. 139, 111-114 (2017).

13. Raymond, J. A. \& DeVries, A. L. Adsorption inhibition as a mechanism of freezing resistance in polar fishes. Proc. Natl Acad. Sci. USA 74, 2589-2593 (1977).

14. Madura, J. D., Baran, K. \& Wierzbicki, A. Molecular recognition and binding of thermal hysteresis proteins to ice. J. Mol. Recognit. 13, 101-113 (2000).

15. Ramakrishnan, S. K. et al. Insights on the facet specific adsorption of amino acids and peptides toward platinum. J. Chem. Inf. Model. 53, 3273-3279 (2013).

16. Dong, L. et al. Facet-specific assembly of proteins on $\mathrm{SrTiO}_{3}$ polyhedral nanocrystals. Sci. Rep. 4, 5084-5089 (2014).

17. Xie, T. et al. Low-index $\mathrm{ZnO}$ crystal plane-specific binding behavior of whole Immunoglobulin G proteins. Langmuir 31, 10493-10499 (2015).

18. Hughes, Z. E., Kochandra, R. \& Walsh, T. R. Facet-specific adsorption of tripeptides at aqueous $\mathrm{Au}$ interfaces: open questions in reconciling experiment and simulation. Langmuir 33, 3742-3754 (2017).

19. Gu, Z. et al. Facet-regulated adhesion of double-stranded DNA on palladium surfaces. Nanoscale 11, 1827-1836 (2019).

20. Li, H., Sun, H. \& Qian, Z. M. The role of the transferrin-transferrin-receptor system in drug delivery and targeting. Trends Pharmacol. Sci. 23, 206-209 (2002).

21. Choi, C. H. J., Alabi, C. A., Webster, P. \& Davis, M. E. Mechanism of active targeting in solid tumors with transferrin-containing gold nanoparticles. Proc. Natl Acad. Sci. USA 107, 1235-1240 (2010).

22. Daniels, T. R. et al. The transferrin receptor and the targeted delivery of therapeutic agents against cancer. BBA-Gen. Subj. 1820, 291-317 (2012).

23. Liu, L. et al. Facet energy and reactivity versus cytotoxicity: the surprising behavior of CdS nanorods. Nano Lett. 16, 688-694 (2016).
24. Eigenheer, R. et al. Silver nanoparticle protein corona composition compared across engineered particle properties and environmentally relevant reaction conditions. Environ. Sci.: Nano 1, 238-247 (2014).

25. Lau, B. L. T. \& Hsu-Kim, H. Precipitation and growth of zinc sulfide nanoparticles in the presence of thiol-containing natural organic ligands. Environ. Sci. Tech. 42, 7236-7241 (2008).

26. Wang, L. et al. Revealing the binding structure of the protein corona on gold nanorods using synchrotron radiation-based techniques: understanding the reduced damage in cell membranes. J. Am. Chem. Soc. 135, 17359-17368 (2013).

27. Huang, X., Hou, X., Song, F., Zhao, J. \& Zhang, L. Facet-dependent Cr(VI) adsorption of hematite nanocrystals. Environ. Sci. Tech. 50, 1964-1972 (2016).

28. Lounsbury, A. W. et al. Preferential adsorption of selenium oxyanions onto $\{110\}$ and $\{012\}$ nano-hematite facets. J. Colloid Interf. Sci. 537, 465-474 (2019).

29. Penna, M. J., Mijajlovic, M. \& Biggs, M. J. Molecular-level understanding of protein adsorption at the interface between water and a strongly interacting uncharged solid surface. J. Am. Chem. Soc. 136, 5323-5331 (2014).

30. $\mathrm{Wu}, \mathrm{X}$.-J. et al. Controlled growth of high-density CdS and CdSe nanorod arrays on selective facets of two-dimensional semiconductor nanoplates. Nat. Chem. 8, 470-475 (2016).

31. Ramalingam, G., Melikechi, N., Christy, P. D., Selvakumar, S. \& Sagayaraj, P. Structural and optical property studies of CdSe crystalline nanorods synthesized by a solvothermal method. J. Cryst. Growth 311, 3138-3142 (2009).

32. Hu, C. et al. Water-induced structure phase transition of CdSe nanocrystals in composite hydroxide melts. Phys. E 42, 1790-1794 (2010).

33. Xiong, S., Xi, B. \& Qian, Y. CdS hierarchical nanostructures with tunable morphologies: preparation and photocatalytic properties. J. Phys. Chem. C. 114, 14029-14035 (2010)

34. Vairavamurthy, A. \& Mopper, K. Field method for determination of traces of thiols in natural waters. Anal. Chim. Acta 236, 363-370 (1990).

35. Kresse, G. \& Hafner, J. Ab initio molecular dynamics for open-shell transition metals. Phys. Rev. B 48, 13115-13118 (1993).

36. Kresse, G. \& Furthmuller, J. Efficient iterative schemes for Ab initio totalenergy calculations using a plane-wave basis set. Phys. Rev. B 54, 11169-11186 (1996).

37. Kresse, G. \& Joubert, D. From ultrasoft pseudopotentials to the projector augmented-wave method. Phys. Rev. B 59, 1758-1775 (1999).

38. Grimme, S. Semiempirical GGA-type density functional constructed with a long-range dispersion correction. J. Comput. Chem. 27, 1787-1799 (2006).

39. Monkhorst, H. \& Pack, J. Special points for brillouin-zone integrations. Phys Rev. B 13, 5188-5192 (1976).

40. Noinaj, N. et al. Structural basis for iron piracy by pathogenic neisseria. Nature 483, 53-58 (2012)

41. Jain, A. et al. Commentary: the materials project: a materials genome approach to accelerating materials innovation. APL Mater. 1, 011002 (2013).

42. Cosseddu, S. \& Infante, I. Force field parametrization of colloidal CdSe nanocrystals using an adaptive rate Monte Carlo optimization algorithm. J. Chem. Theory Comput. 13, 297-308 (2017)

43. Abraham, M. J. et al. Gromacs: high performance molecular simulations through multi-level parallelism from laptops to supercomputers. SoftwareX 1-2, 19-25 (2015).

44. Pronk, S. et al. Gromacs 4.5: a high-throughput and highly parallel open source molecular simulation toolkit. Bioinformatics 29, 845-854 (2013).

45. Van Der Spoel, D. et al. Gromacs: fast, flexible, and free. J. Comput. Chem. 26, 1701-1718 (2005)

46. Lindorff-Larsen, K. et al. Improved side-chain torsion potentials for the mber Ff99SB protein force field. Proteins 78, 1950-1958 (2010).

47. Evans, D. J. \& Holian, B. L. The Nose-Hoover thermostat. J. Physic. Chem. 83, 4069-4074 (1985).

48. Darden, T., York, D. \& Pedersen, L. Particle mesh Ewald: an N.log(N) method for Ewald sums in large systems. J. Physic. Chem. 98, 10089-10092 (1993).

49. Hess, B., Bekker, H., Berendsen, H. J. C. \& Fraaije, J. G. E. M. LINCS: a linear constraint solver for molecular simulations. J. Comput. Chem. 18, 1463-1472 (1997).

50. Jorgensen, W. L., Chandrasekhar, J., Madura, J. D., Impey, R. W. \& Klein, M. L. Comparison of simple potential functions for simulating liquid water. J. Physic. Chem. 79, 926-935 (1983).

51. Humphrey, W., Dalke, A. \& Schulten, K. Visual molecular dynamics. J. Mol. Graph. 14, 33-38 (1996).

\section{Acknowledgements}

This project was supported by the National Natural Science Foundation of China (Grants 21976095 and 21425729), Tianjin Municipal Science and Technology Commission (17JCYBJC23100), and the 111 Program of the Ministry of Education of China (T2017002). Partial funding for P.J.J.A. was provided by the NSF ERC for Nanotechnology-Enabled Water Treatment (EEC-1449500). We thank Lu Liu and Xue Chen for assistance with nanomaterial preparation, Haijun Zhang and Weichao Wang 
for helping with theoretical calculations, and Ligang $\mathrm{Hu}$, Guangbo Qu, and Qilin Yu for helpful discussion regarding proteomics.

\section{Author contributions}

Y.Q. carried out all experiments and data analysis. T.Z., W.C., and P.J.J.A. conceived the study and supervised the research. C.J., S.L., and C.Z. contributed intellectual input to this study, and Y.Q., T.Z., P.J.A., and W.C. drafted the paper.

\section{Competing interests}

The authors declare no competing interests.

\section{Additional information}

Supplementary information is available for this paper at https://doi.org/10.1038/s41467020-14972-z.

Correspondence and requests for materials should be addressed to T.Z. or P.J.J.A.

Peer review information Nature Communications thanks the anonymous reviewers for their contribution to the peer review of this work. Peer reviewer reports are available.
Reprints and permission information is available at http://www.nature.com/reprints

Publisher's note Springer Nature remains neutral with regard to jurisdictional claims in published maps and institutional affiliations.

(c) Open Access This article is licensed under a Creative Commons Attribution 4.0 International License, which permits use, sharing, adaptation, distribution and reproduction in any medium or format, as long as you give appropriate credit to the original author(s) and the source, provide a link to the Creative Commons license, and indicate if changes were made. The images or other third party material in this article are included in the article's Creative Commons license, unless indicated otherwise in a credit line to the material. If material is not included in the article's Creative Commons license and your intended use is not permitted by statutory regulation or exceeds the permitted use, you will need to obtain permission directly from the copyright holder. To view a copy of this license, visit http://creativecommons.org/ licenses/by/4.0/.

(C) The Author(s) 2020 